\title{
Tienilic acid: a single treatment for hyperuricaemia and hypertension?
}

\author{
T. GIBSON, V. A. RODGERS, C. F. POTTER, H. A. SIMMONDS
}

From the Guy's Arthritis Research Unit, and the Department of Medicine, Guy's Hospital Medical School, London SE1 9RT

SUMMARY Tienilic acid is a drug with established uricosuric and hypotensive properties. We have examined its potential role as a single treatment for hyperuricaemia and hypertension, 2 disorders which are commonly associated. In 17 subjects with gout, blood uric acid levels were reduced by approximately $50 \%$. Eleven of these patients also had hypertension which was improved by tienilic acid. However, a statistically significant effect was observed only with standing diastolic blood pressure. Side effects included acute episodes of gout in 4 patients and transient loin pain and dysuria in 1 patient. The precipitation of gouty arthritis is an acknowledged risk of all potent hypouricaemic drugs. The hazard of urate deposition in the renal tract implicit in the history of loin pain is a more serious complication. Thus, it would appear that tienilic acid is a potentially valuable drug which may have a special role in patients with hyperuricaemia and hypertension. Further study is necessary to determine how it may be best administered without the risk of renal damage.

A relationship between hyperuricaemia and hypertension has been observed in population surveys, ${ }^{12}$ and in studies confined to both hypertensive ${ }^{3}$ and gouty subjects. ${ }^{4}$ When these 2 disorders coincide, diuretic therapy for hypertension may be injudicious because it may accentuate hyperuricaemia unless a concurrent hypouricaemic agent is prescribed.

Tienilic acid is a new diuretic with hypotensive and marked hypouricaemic properties. ${ }^{5}$ For this reason it has been proposed as the drug of first choice in patients with hypertension who already have gout or are likely to develop it when taking thiazide diuretics. ${ }^{6}$ The study reported here was an attempt to determine whether tienilic acid alone may be adequate treatment for the hyperuricaemia of gouty patients and to guage its effect on the hypertension which may be associated with gout.

\section{Patients and methods}

Seventeen male patients with a history of recurrent acute gout and sustained hyperuricaemia entered the study, which was conducted on an outpatient basis. Their mean age was 54 (range 31-69) years. Eleven

Accepted for publication 15 April 1980

Correspondence to Dr T. Gibson, Department of Rheumatology, Guy's Hospital, London SE1 9RT. subjects had acknowledged mild hypertension (recumbent diastolic) $>95 \mathrm{mmHg}$ ) which was being treated prior to the study with miscellaneous hypotensive drugs. All patients gave their written consent to the study, which was explained to each participant in detail.

One week before the initial assessments the patients discontinued their usual hypotensive and hypouricaemic medicines. Prophylactic colchicine, $0.5 \mathrm{mg}$ twice daily, was substituted and continued for the duration of the study. Clinical and laboratory measurements were repeated 1 week after the initial evaluation and immediately before beginning treatment with tienilic acid $125 \mathrm{mg}$ twice daily. Further assessments were performed 2 and 4 weeks after commencing treatment. No amendments were made to the patients' usual diets.

At each attendance subjects were weighed and blood pressure was recorded after the 5 minutes recumbency and 2 minutes standing. Diastolic blood pressure was measured at the point where sounds were muffled (Korotkov IV). Blood samples were obtained after an overnight fast for sodium, potassium, bicarbonate, calcium, phosphate, urea, creatinine, triglyceride, cholesterol, glucose, and uric acid measurements. Haemoglobin, packed cell volume, white cell, and platelet counts were 
performed at the same time. Before each assessment $24 \mathrm{~h}$ urine samples were collected under toluene and paraffin for estimation of $\mathrm{pH}$, sodium, potassium, calcium, phosphate, creatinine, protein, uric acid, ammonium, and titratable acid. Blood and urine electrolytes, creatinine, urea, glucose and lipids were measured by standard AutoAnalyzer techniques. Urine titratable acid and ammonium were determined by the method of $\mathrm{Chan}^{7}$ and all uric acid values by the uricase method of Simmonds. ${ }^{8}$ The latter method gives results for uric acid which in general are lower than those achieved by the uricase method adapted for the AutoAnalyzer. Two consecutive $24 \mathrm{~h}$ urine collections were obtained for the evaluation at weeks 2 and 4 and their mean results used in the analysis. The mean measurement from each assessment were compared statistically by Student's $t$ test.

\section{Results}

Attacks of gout occurred in 4 patients at various times during the treatment period. This necessitated withdrawal of 1 patient in the third week, and therefore the observations at week 4 refer to only 16

Table 1 Mean $\pm S D$ systolic and diastolic blood pressure of 11 hypertensive patients before and after treatment with tienilic acid

\begin{tabular}{llcccc}
\hline $\begin{array}{l}\text { Time } \\
\text { (weeks) }\end{array}$ & & \multicolumn{2}{c}{ Control period } & \multicolumn{2}{c}{ Tienilic acid treatment } \\
\cline { 2 - 6 } & & -1 & 0 & 2 & 4 \\
\hline Supine & Systolic & $149 \pm 18$ & $142 \pm 16$ & $131 \pm 13$ & $137 \pm 18$ \\
(mmHg) & Diastolic & $97 \pm 9$ & $95 \pm 13$ & $90 \pm 7$ & $88 \pm 8$ \\
Standing & Systolic & $147 \pm 15$ & $141 \pm 15$ & $131 \pm 8$ & $133 \pm 14$ \\
(mmHg) & Diastolic & $103 \pm 6$ & $102 \pm 7$ & $96 \pm 4^{*}$ & $94 \pm 9^{*}$ \\
\hline
\end{tabular}

*P<0.05 compared with week 0 . subjects. One patient noted transient loin pain and dysuria at the onset of his acute gout during the second week of treatment. Examination of a midstream urine specimen $24 \mathrm{~h}$ after this event was normal, as were blood urea and creatinine estimations. He was allowed to complete the study taking tienilic acid $125 \mathrm{mg}$ daily, and, since the reduced dose achieved a biochemical response which was consistent with the overall trend, the results were included in the final analysis. No other side effects were recorded apart from mild headache in 2 patients None developed proteinuria.

Insignificant reductions of mean supine and standing blood pressure were seen in the patients as a whole. A more pronounced decline of mean blood pressure was observed in those with hypertension (Table 1) and this was significant for standing diastolic values at week $2(t=2.21 ; \mathrm{P}<0.05)$ and week 4 $(t=2 \cdot 1 ; \mathrm{P}<0.05)$.

Table 2 outlines the results of blood investigations before and during treatment. Plasma uric $\stackrel{0}{\mathscr{9}}$ acid levels declined dramatically and significantly at week $2(t=8.63 ; \mathrm{P}<0.001)$ and week $4(t=6 \cdot 12$; $\mathbf{P}<0.001)$. Blood urea and creatinine rose slightly and serum potassium fell significantly by week 2 $(t=4 \cdot 72 ; \mathrm{P}<0 \cdot 001)$ and week $4(t=4 \cdot 04 ; \mathrm{P}<0 \cdot 001)$. Serum calcium was elevated significantly at week 4 $(t=2 \cdot 1 ; \mathrm{P}<0.05)$.

Body weight and serum sodium, bicarbonate, phosphate, triglyceride, cholesterol, and glucose did not alter significantly. There was no change of haemoglobin, packed cell volume, white cell, or platelet counts.

Mean urine excretion and clearance values are detailed in Table 3. Urate excretion increased during tienilic acid treatment and this achieved significance at week $4(t=2 \cdot 86 ; \mathrm{P}<0 \cdot 01)$. Urate clearance was

Table 2 Mean $\pm S D$ body weight and measurements of blood values before and after treatment with tienilic acid. Except where indicated values are expressed in $\mathrm{mmol} / \mathrm{l}$

\begin{tabular}{|c|c|c|c|c|}
\hline \multirow[t]{2}{*}{ Time (weeks) } & \multicolumn{2}{|c|}{ Control period } & \multicolumn{2}{|c|}{ Tienilic acid treatment } \\
\hline & -1 & 0 & 2 & 4 \\
\hline $\begin{array}{l}\text { Body weight (kg) } \\
\text { Uric acid } \\
\text { Urea } \\
\text { Creatinine ( } \mu \mathrm{mol} / \mathrm{l}) \\
\text { Sodium } \\
\text { Potassium } \\
\text { Bicarbonate } \\
\text { Calcium } \\
\text { Phosphate } \\
\text { Triglyceride } \\
\text { Cholesterol } \\
\text { Glucose } \\
\text { Haemoglobin (g/dl) } \\
\text { Packed cell volume }\end{array}$ & $\begin{array}{c}86 \pm 13 \\
0 \cdot 41 \pm 0 \cdot 08 \\
6 \cdot 1 \pm 1 \cdot 7 \\
100 \pm 19 \\
139 \pm 3 \cdot 0 \\
4 \cdot 1 \pm 0 \cdot 4 \\
29 \pm 4 \cdot 0 \\
2 \cdot 37 \pm 0 \cdot 1 \\
0 \cdot 96 \pm 0 \cdot 17 \\
2 \cdot 5 \pm 0 \cdot 9 \\
6 \cdot 7 \pm 1 \cdot 3 \\
4 \cdot 8 \pm 0 \cdot 6 \\
15 \cdot 4 \pm 0 \cdot 9 \\
44 \pm 2 \cdot 3\end{array}$ & $\begin{array}{c}86 \pm 12 \\
0.41 \pm 0 \cdot 08 \\
5 \cdot 8 \pm 1 \cdot 2 \\
99 \pm 24 \\
141 \pm 3 \cdot 5 \\
4 \cdot 0 \pm 0 \cdot 3 \\
27 \pm 4 \cdot 0 \\
2 \cdot 35 \pm 0 \cdot 1 \\
0 \cdot 97 \pm 0 \cdot 26 \\
2 \cdot 3 \pm 1 \cdot 3 \\
6 \cdot 6 \pm 1 \cdot 4 \\
4 \cdot 7 \pm 0 \cdot 7 \\
14 \cdot 9 \pm 0 \cdot 9 \\
43 \pm 1 \cdot 4\end{array}$ & $\begin{array}{c}86 \pm 13 \\
0 \cdot 18 \pm 0 \cdot 05 \dagger \\
6 \cdot 6 \pm 1 \cdot 6 \\
103 \pm 22 \\
141 \pm 4 \cdot 0 \\
3 \cdot 5 \pm 0 \cdot 2 \dagger \\
28 \pm 4 \cdot 7 \\
2 \cdot 42 \pm 0 \cdot 1 \\
0 \cdot 92 \pm 0 \cdot 11 \\
2 \cdot 6 \pm 1 \cdot 1 \\
6 \cdot 5 \pm 1 \cdot 0 \\
4 \cdot 7 \pm 0 \cdot 9 \\
14 \cdot 8 \pm 1 \cdot 0 \\
42 \pm 3 \cdot 0\end{array}$ & $\begin{array}{c}85 \pm 14 \\
0.22 \pm 0.08 \dagger \\
6 \cdot 6 \pm 1 \cdot 7 \\
104 \pm 23 \\
140 \pm 2 \cdot 7 \\
3 \cdot 5 \pm 0 \cdot 5 \dagger \\
28 \pm 4 \cdot 0 \\
2 \cdot 43 \pm 0 \cdot 08^{*} \\
0 \cdot 94 \pm 0 \cdot 1 \\
2 \cdot 8 \pm 1 \cdot 3 \\
6 \cdot 9 \pm 1 \cdot 2 \\
5 \cdot 0 \pm 0 \cdot 8 \\
15 \cdot 2 \pm 0.7 \\
44 \pm 2 \cdot 0\end{array}$ \\
\hline
\end{tabular}

$* \mathrm{P}<0.05 ; \nmid \mathrm{P}<0.001$ compared with week 0 . 
Table 3 Mean $\pm S D$ urine and clearance values before and after treatment with tienilic acid. The figures in the table represent urinary excretion levels in $\mathrm{mmol} / 24 \mathrm{~h}$

\begin{tabular}{|c|c|c|c|c|}
\hline \multirow[t]{2}{*}{ Time (weeks) } & \multicolumn{2}{|c|}{ Control period } & \multicolumn{2}{|c|}{ Tienilic acid treatment } \\
\hline & -1 & 0 & 2 & 4 \\
\hline $\begin{array}{l}\text { Uric acid } \\
\text { Sodium } \\
\text { Potassium } \\
\text { Calcium } \\
\text { Phosphate } \\
\text { pH } \\
\text { Ammonium } \\
\text { Titratable acid } \\
\text { Urate clearance }(\mathrm{ml} / \mathrm{min}) \\
\text { Creatinine clearance }(\mathrm{ml} / \mathrm{min})\end{array}$ & $\begin{aligned} 2 \cdot 7 & \pm 0 \cdot 9 \\
149 & \pm 59 \\
52 & \pm 19 \\
4 \cdot 9 & \pm 1 \cdot 7 \\
25 \cdot 6 & \pm 7 \cdot 8 \\
5 \cdot 3 & \pm 0 \cdot 4 \\
37 & \pm 11 \\
22 & \pm 9 \cdot 0 \\
4 \cdot 8 & \pm 2 \cdot 0 \\
80 & \pm 21\end{aligned}$ & $\begin{array}{l}2 \cdot 9 \pm 1 \cdot 0 \\
156 \pm 61 \\
56 \pm 24 \\
5 \cdot 1 \pm 2 \cdot 0 \\
27 \cdot 8 \pm 10 \cdot 9 \\
5 \cdot 4 \pm 0 \cdot 3 \\
38 \pm 12 \\
22 \pm 11 \\
5 \cdot 0 \pm 1 \cdot 8 \\
91 \pm 27\end{array}$ & $\begin{array}{l}3 \cdot 7 \pm 1 \cdot 4 \\
162 \pm 64 \\
59 \pm 20 \\
4 \cdot 1 \pm 2 \cdot 0^{*} \\
28 \pm 8 \cdot 8 \\
5 \cdot 3 \pm 0 \cdot 2 \\
35 \pm 11 \\
24 \pm 12 \cdot 5 \\
15 \cdot 0 \pm 7 \cdot 1 \dagger \\
88 \pm 21\end{array}$ & $\begin{array}{l}4 \cdot 2 \pm 1 \cdot 6^{* *} \\
181 \pm 82 \\
68 \pm 25 \\
4 \cdot 7 \pm 3 \cdot 1 \\
29 \pm 11 \\
5 \cdot 4 \pm 0 \cdot 4 \\
37 \pm 18 \\
26 \pm 12 \\
13 \cdot 7 \pm 6 \cdot 6 \dagger \\
82 \pm 21\end{array}$ \\
\hline
\end{tabular}

${ }^{*} \mathbf{P}<0.05 ;{ }^{* *} \mathrm{P}<0.01 ; \dagger<0.001$ compared with week 0 .

increased commensurate with the decline of blood uric acid and was significant at week $2(t=5 \cdot 6$; $\mathrm{P}<0 \cdot 001)$ and week $4(t=5 \cdot 29 ; \mathrm{P}<0 \cdot 001)$. Sodium and potassium excretion were both increased by tienilic acid, but the difference between mean values did not reach the conventional level of statistical significance. By contrast, urine calcium fell significantly at week $2(t=2 \cdot 42 ; \mathrm{P}<0.05)$.

There was no change in the mean urine excretion of phosphate, ammonium, and titratable acid nor of urine $\mathrm{pH}$ or creatinine clearance.

\section{Discussion}

We have confirmed the potent uricosuric property of tienilic acid previously observed by others. ${ }^{56}$ The mean blood uric acid levels of the gout patients was reduced by approximately $50 \%$. Tienilic acid is therefore sufficiently hypouricaemic to warrant consideration as a new treatment for hyperuricaemia. Its efficacy in this regard now requires comparison with other blood uric acid lowering drugs.

The mean systolic and diastolic blood pressure of 11 hypertensive gouty patients declined during tienilic acid treatment. However, statistically significant reductions were observed only for standing diastolic blood pressure values. These findings need to be tempered by the nature of the trial, which was conducted in an open fashion and without incorporation of a placebo control treatment. The observations are nevertheless in accord with those of previous studies which have demonstrated that tienilic acid has hypotensive properties which are equivalent to bendrofluazide ${ }^{6}$ and additive to those of propranolol. ${ }^{9}$

Tienilic acid was associated with minor elevation of blood urea and creatinine, but creatinine clearance was not altered. These findings are consistent with previous reports. ${ }^{59}$ Serum sodium did not fall despite a modest natriuretic response. An increase of potassium excretion was reflected in a significant reduction of serum potassium, an effect which in an earlier study was similar to that of hydrochlorothiazide. ${ }^{5}$ An increase of serum calcium was associated with the acknowledged hypocalciuric action of tienilic acid, ${ }^{5}$ another property which it shares with thiazide diuretics. ${ }^{10}$ Phosphate excretion was unaffected.

Fasting blood glucose and lipids were not influenced by tienilic acid, and we were unable to confirm a reduction of serum triglyceride.11 There was no effect on the haematological picture and in particular we did not observe any fall of haemoglobin concentrations as noted by Pearson et al. ${ }^{9}$

Attacks of gout seen in 4 patients reflect the well known risks associated with drugs which are able to alter blood uric acid levels abruptly. Of more concern was the transient episode of dysuria and loin pain seen in 1 patient. This was presumptive but unconfirmed evidence of uric acid precipitation in the renal tract. A drug which is as profoundly uricosuric as tienilic acid will invariably accentuate the risk of uric acid crystal deposition in the renal collecting ducts, especially in hyperuricaemic patients.

No reduction of urine $\mathrm{pH}$ or change in the pattern of hydrogen ion excretion was demonstrated in this study, and thus urate crystallisation was not made more likely by a lowered urine $\mathrm{pH}$. Acute renal failure during tienilic acid therapy has been recently reported, and the speculative cause was acute urate nephropathy. ${ }^{12}{ }^{13}$ It remains to be seen whether this serious hazard may be obviated by ensuring adequate hydration before beginning treatment or by the administration of an initially smaller dose of tienilic acid.

Further study of this effective and potentially valuable uricosuric and hypotensive drug is warranted. In particular, there is a need to determine the dose and conditions which will lessen the risk of renal damage. 
We are most grateful for the generous assistance and cooperation provided by Smith Kline and French Ltd.

NOTE:-Readers are referred to an Addendum to the previous paper by J. A. Reardon and J. T. Scott.

\section{References}

1 Klein R, Klein B E, Cornoni J C, Maready J, Cassel J C, Tyroler H A. Serum uric acid. Its relationship to coronary heart disease risk factors and cardiovascular disease, Evans County, Georgia. Arch Intern Med 1973; 132: 401-10.

2 Yano K, Rhoads G G, Kagan A. Epidemiology of serum uric acid among 8,000 Japanese-American men in Hawaii. J Chron Dis 1977; 30: 171-84.

3 Cannon P J, Stason W B, De Martini F E, Sommers S C, Laragh $J$ H. Hyperuricaemia in primary and renal hypertension. $N$ Engl J Med 1966; 275: 457-64.

4 Grahame R, Scott J T. Clinical survey of 354 patients with gout. Ann Rheum Dis 1970; 29: 461-8.

5 Lemieux G, Beauchemin M, Gougoux A, Vinay $P$. Treatment of arterial hypertension with tienilic acid, a new diuretic with uricosuric properties. Can Med Assoc $J$ 1978; 118: 1074-80.
- Roberts C J C, Marshall A J, Heaton S, Barritt D W, Comparison of natriuretic uriccsuric and antihyper- $\vec{S}$ tensive properties of tienilic acid, bedrofluazide and 7 spironolactone. $\mathrm{Br}$ Med J 1979; i: 224-6.

7 Chan J C M. The rapid determination of urinary titra- $\overline{\bar{\omega}}$ table acid and ammonium and evalauation of freezing as a method of preservation. Clin Biochem 1972; 5: 94-8. ¿

8 Simmonds H A. A method of estimation of uric acid in urine and other body fluids. Clin Chim Acta 1967; 15: 375-8.

9 Pearson R M, Bulpitt C J, Havard C W H. Biochemical and haematological changes induced by tienilic acid $\vec{\omega}$ combıned with propanolol in essential hypertension. Lancet 1979; i: 697-9.

10 Goulding A, Spears G F, Simpson F O. Effects of different diuretics on urinary calcium excretion in a general population. Aust $N Z$ J Med 1978; 8: 474-8.

11 Brooks C S, Marx B. Hypolipemic effect of the diuretic $\omega$ ticrynafen. Kidney Int 1977; 12 : 496.

12 Bennett W M, Van Zee B E. Acute renal failure from ticrynafen, $N$ Engl J Med 1979; 301 : 1179-80.

13 Selby $T$. Acute renal failure from ticrynafen. $N$ Engl J Med 1979; 301 : 1180-1. 